\title{
Visualization of Activated Platelets by Targeted Magnetic Resonance Imaging Utilizing Conformation-Specific Antibodies against Glycoprotein IIb/IIla
}

\author{
Constantin von zur Muhlen ${ }^{\mathrm{a}, \mathrm{b}}$ Karlheinz Peter ${ }^{\mathrm{c}}$ Ziad A. Ali ${ }^{\mathrm{a}}$ \\ Jürgen E. Schneider ${ }^{a}$ Martina A. McAteer ${ }^{a}$ Stefan Neubauer ${ }^{a}$ \\ Keith M. Channon ${ }^{a}$ Christoph Bode ${ }^{b}$ Robin P. Choudhury ${ }^{a}$ \\ ${ }^{a}$ Department of Cardiovascular Medicine, University of Oxford, Oxford, UK; ${ }^{b}$ Department of Cardiology and \\ Angiology, University of Freiburg, Freiburg, Germany; ${ }^{\complement}$ Center for Thrombosis and Myocardial Infarction, \\ Baker Heart Research Institute, Melbourne, Australia
}

\section{Key Words}

Atherosclerosis • Magnetic resonance imaging •

Molecular imaging $\cdot$ Platelets $\cdot$ Thrombosis

\begin{abstract}
Ruptured atherosclerotic plaques, lined with activated platelets, constitute an attractive target for magnetic resonance imaging (MRI). This study evaluated whether microparticles of iron oxide (MPIO) targeting ligand-induced binding sites (LIBS) on the activated conformation of glycoprotein Ilb/llla could be used to image platelets. MPIO (size: $1 \mu \mathrm{m}$ ) were conjugated to anti-LIBS or control single-chain antibody. Following guidewire injury to mouse femoral artery, platelet adhesion was present after $24 \mathrm{~h}$. Mice were perfused with anti-LIBS-MPIO (or control MPIO) via the left ventricle and 11.7-tesla MRI was performed on femoral arteries ex vivo. A $3 \mathrm{D}$ gradient echo sequence attained an isotropic resolution of $25 \mu \mathrm{m}$. MPIO binding, quantified by MRI, was 4-fold higher with anti-LIBS-MPIO in comparison to control MPIO $(p<$ $0.01)$. In histological sections, low signal zones on MRI and MPIO correlated strongly $\left(R^{2}=0.72 ; p<0.001\right)$, indicating accurate MR quantification. In conclusion, anti-LIBS-MPIO bind to activated platelets in mouse arteries, providing a basis for
\end{abstract}

the use of function-specific single-chain antibody-MPIO conjugates for molecular MRI, and represent the first molecular imaging of a conformational change in a surface receptor. This presents an opportunity to specifically image activated platelets involved in acute atherothrombosis with MRI.

Copyright $\odot 2008$ S. Karger AG, Basel

\section{Introduction}

Activated platelets adhere and aggregate rapidly at the site of arterial injury [1] and contribute to the pathogenesis of acute coronary syndromes [2]. Anti-platelet agents are effective in reducing complications in a proportion of patients with acute ST-segment elevation myocardial infarction (MI) [3, 4], non-ST-segment elevation MI [5] and in the context of acute and high-risk percutaneous coronary intervention [6-8]. However, the relative contribution of platelets varies in individual patients and according to clinical circumstances [9]. Therefore, precise deter-

C. von zur Muhlen and K. Peter contributed equally to the study.

\section{KARGER}

Fax +41613061234 E-Mail karger@karger.ch www.karger.com (c) 2008 S. Karger AG, Basel

1018-1172/09/0461-0006\$24.50/0

Accessible online at:

www.karger.com/jvr
Dr. Robin Choudhury

Department of Cardiovascular Medicine, John Radcliffe Hospita

Oxford OX3 9DU (UK)

Tel. +44 1865221 870, Fax +441865740352

E-Mail robin.choudhury@cardiov.ox.ac.uk 
mination of the presence and location of activated platelets would greatly aid in diagnosis and, potentially, provide the basis for an optimal choice of therapy.

Recent progress in magnetic resonance imaging (MRI) has allowed detailed non-invasive determination of atherosclerotic plaque composition and morphology [10]. There is currently considerable interest in developing contrast ligand probes to enable imaging of specific molecules, cells and processes involved in atherothrombosis $[10,11]$. Fibrin-rich thrombi have been targeted with paramagnetic nanoparticles conjugated to anti-fibrin $\mathrm{F}(\mathrm{ab})^{\prime}$ fragments [12] and with a fibrin-specific small molecule-gadolinium chelate [13]. Platelet-rich thrombi can be identified by coronary angioscopy [9]. Recently, exogenously labeled platelets have been imaged in surgically exposed large arteries using near infra-red microscopy in an animal model [14]. However, there is currently no means to image platelets using non-invasive modalities, such as MRI.

Shapiro et al. [15] recently described the use of microparticles of iron oxide (MPIO) for cellular imaging and tracking. These MPIO convey a payload of iron that is many orders of magnitude greater than iron nanoparticles (ultrasmall particles of iron oxide) and cause local magnetic field inhomogeneity extending for a distance $\sim 50$ times the physical diameter of the microparticle. We therefore hypothesized that with a suitable ligand, MPIO could be used for molecular imaging of activated platelets. The platelet integrin GPIIb/IIIa $\left(\alpha_{\mathrm{IIb}} \beta_{3}, \mathrm{CD} 41 / \mathrm{CD} 61\right)$ mediates the final common pathway of platelet aggregation and is the key to thrombus formation on ruptured atherosclerotic plaques. We have recently developed a single-chain antibody that recognizes ligand-induced binding sites (LIBS) of GPIIb/IIIa that become exposed only upon receptor activation and ligand binding $[16,17]$. The LIBS epitope is located outside the fibrinogen binding area and therefore yields high specificity for the activated GPIIb/IIIa receptor. Consequently, it can also be targeted even in the presence of fibrinogen or GPIIb/IIIa receptor antagonists. In contrast to non-activation-specific anti-GPIIb/IIIa antibodies, such as abciximab, the anti-LIBS single-chain antibody does not bind to circulating platelets. It only binds to platelets which are activated by adhesion to inflamed or injured vessel walls or to activated platelets that are part of platelet aggregates [16]. Given its specificity, the anti-LIBS single-chain antibody presents a compelling tool for molecular imaging of activated platelets with MRI.

Accordingly, we have developed a contrast agent consisting of anti-LIBS single-chain antibodies conjugated to
MPIO and designed to target activated platelets. We report here the capability of this contrast agent to identify activated platelets in a mouse model of endothelial injury [18] and accurate identification of MPIO binding using high-resolution ex vivo MRI.

\section{Methods}

Single-Chain Antibody Generation and Conjugation to

1- $\mu$ m Iron Oxide Microparticles

The monoclonal antibody anti-LIBS 145 binds to GPIIb/IIIa only in its active conformation and exhibits strong binding to ADP-activated platelets in the presence of fibrinogen [17]. The monoclonal antibody anti-LIBS 145 expressing hybridoma cell line was used as the basis for cloning of an anti-LIBS single-chain antibody (scFv). mRNA of this hybridoma cell line was prepared and reverse transcribed using an oligo-dT primer. The variable regions of the heavy and light chain of the antibody were amplified using PCR primers that anneal to conserved regions at the $5^{\prime}$ and $3^{\prime}$ ends of the variable regions. The PCR products were cloned into the pHOG21 vector, TG1 Escherichia coli were transformed, and individual clones were assessed for LIBS-typical binding to GPIIb/IIIa in flow cy tometry using activated platelets. Finally, the best binding $\mathrm{scFv}_{\text {LIBS }}$ was produced in LB media containing 100 $\mu \mathrm{g} / \mathrm{ml}$ ampicillin and $100 \mathrm{~mm}$ glucose at $37^{\circ} \mathrm{C}$ in $500-\mathrm{ml}$ flasks. Cultures were shaken at $200 \mathrm{rpm}$ for approximately $4-6 \mathrm{~h}$ until an $\mathrm{OD}(600 \mathrm{~nm})$ of $\sim 0.8$ was reached. Bacteria were pelleted by centrifugation at $5,000 \mathrm{rpm}$ for $10 \mathrm{~min}$ at $4^{\circ} \mathrm{C}$ and resuspended with LB media containing $100 \mu \mathrm{g} / \mathrm{ml}$ ampicillin and $0.4 \mathrm{M}$ sucrose (both Sigma, Deisenhofen, Germany). IPTG was added to a final concentration of $0.25 \mathrm{~mm}$ for induction of $\mathrm{scFv}$ production and incubated at room temperature $\left(22-24^{\circ} \mathrm{C}\right)$ with $200 \mathrm{rpm}$ for $16-$ $20 \mathrm{~h}$. Bacteria were harvested by centrifugation at 5,000 rpm for $10 \mathrm{~min}$ at $4^{\circ} \mathrm{C}$, the pelleted bacteria were resuspended in $5 \mathrm{ml} 1 \times$ BugBuster $^{\circledR}$ (Novagen, Freiburg, Germany) solution/g pellet and incubated for $15 \mathrm{~min}$ at room temperature with gentle shaking. After an additional centrifugation step at 15,000 rpm for $20 \mathrm{~min}$ at $4^{\circ} \mathrm{C}$, the supernatant containing soluble protein was kept on ice and a protease inhibitor (Complete ${ }^{\circledR}$; Roche, Mannheim, Germany) diluted 1:50 was added. The supernatant was mixed with 500 $\mu \mathrm{l} \mathrm{Ni}{ }^{2+}$ agarose (Qiagen, Hilden, Germany) and incubated for $1 \mathrm{~h}$ at $4{ }^{\circ} \mathrm{C}$ with constant shaking at $150 \mathrm{rpm}$. $\mathrm{Ni}^{2+}$ agarose, now binding His-tagged proteins, was allowed to settle for $30 \mathrm{~min}$ before being washed with buffer $\left(50 \mathrm{mM} \mathrm{NaH}_{2} \mathrm{PO}_{4}, 300 \mathrm{mM} \mathrm{NaCl}\right.$ and $20 \mathrm{~mm}$ imidazole, $\mathrm{pH}$ 8). This batch washing procedure was repeated twice. Finally, the scFv was eluted at high imidazole concentrations $(250 \mathrm{mM})$ and finally dialyzed to PBS. Functionality of the scFv preparations was evaluated in flow cytometry.

Cobalt-functionalized MPIO $(1 \mu \mathrm{m})$ were conjugated to the histidine tag of the LIBS single-chain antibody referring to the protocol of the manufacturer (Dynal-Beads TALON, Dynal Biotech, Oslo, Norway; www.invitrogen.com/dynal). In brief, $1 \mathrm{mg}$ of MPIO was incubated with the LIBS antibody for $10 \mathrm{~min}$ at room temperature to bind approximately $10 \mu \mathrm{g}$ of histidine-tagged antibody. The tube containing the suspension was then placed on a magnet until the MPIO had migrated to the side of the tube, and the supernatant was discarded. This washing was repeated four 
times using a binding and washing buffer containing $50 \mathrm{mM} \mathrm{NaP}$ (pH 8), $300 \mathrm{~mm} \mathrm{NaCl}$ and $0.01 \%$ Tween-20.

\section{LIBS-MPIO Binding to Activated Platelets}

An adhesion assay was performed to demonstrate binding of the LIBS-MPIO to activated platelets. Since MPIO are visualized by light microscopy, they played a role analogous to that of the secondary antibody in immunohistochemistry. Blood from a healthy volunteer taking no medication was anticoagulated with citric acid and centrifuged at 1,000 rpm for $10 \mathrm{~min}$. The resulting platelet-rich plasma was diluted with PBS (1:10) and $100 \mu$ l were added onto fibrinogen-covered coverslips, which had been preincubated with $20 \mu \mathrm{g} / \mathrm{ml}$ fibrinogen for $1 \mathrm{~h}$ at $37^{\circ} \mathrm{C}$ and blocked with $1 \%$ bovine serum albumin (Sigma) for $1 \mathrm{~h}$ at room temperature. After a $30-\mathrm{min}$ incubation at $37^{\circ} \mathrm{C}$, coverslips were washed with $\mathrm{PBS}$ and under continuous rotation either incubated with 0.5 $\mu \mathrm{g}$ of LIBS-MPIO or an equivalent conjugated irrelevant singlechain antibody control (control MPIO) for another $30 \mathrm{~min}$ at $37^{\circ} \mathrm{C}$. Coverslips were then washed twice for $5 \mathrm{~min}$ with PBS and blocked with $10 \%$ goat serum (Vector, Burlingame, Calif., USA) for $1 \mathrm{~h}$ at room temperature. Due to red autofluorescence of the MPIO, LIBS-MPIO and control MPIO contrast agent could be readily identified by confocal microscopy. To demonstrate specific binding of the contrast agent, platelets were co-stained for P-selectin using a monoclonal mouse anti-human CD62 antibody (1:100, R\&D Systems, Abingdon, UK) with a biotinylated goat anti-mouse IgG (Vector, Burlingame, Calif., USA) serving as a secondary antibody. Finally, fluorescein avidin D (Vector) diluted 1:200 was added and incubated for $1 \mathrm{~h}$ at room temperature. Coverslips were fixed using CellFix (BD Biosciences, Heidelberg, Germany) and evaluated by confocal microscopy.

\section{Mice}

Wire injury was performed in male C57BL/6 mice (Jackson Laboratories, Margate, UK), mean age $10 \pm 0.8$ weeks. Mice received water and standard chow diet ad libitum. All procedures were performed in accordance with the UK Home Office Animals (Scientific Procedures) Act 1986.

\section{Femoral Wire Injury, MPIO Perfusion and Sample}

Preparation ex vivo

Single-sided femoral wire injury was performed under general anesthesia in 13 mice, using a combination of Hypnorm $(25 \mathrm{mg} / \mathrm{kg}$, Bayer, Leverkusen, Germany) and Hypnoval (25 mg/kg, Bayer) administered subcutaneously, as described previously [18]. Under a surgical microscope, a groin incision was made. The femoral artery was exposed, and an arteriotomy was made distal to the epigastric branch using a 30-gauge injection cannula (BD, Erembodegem, Belgium). A 0.010' guidewire (Boston Scientific, Natick, Mass., USA) was inserted, advanced to the aortic bifurcation, and pulled back. After removal of the wire, the arteriotomy site was ligated and the skin closed using silk sutures. After $24 \mathrm{~h}$, mice were terminally anesthetized by inhalation of isoflurane. The chest was opened by thoracotomy, the heart exposed and the right atrium cut. A 30 gauge needle was inserted through the apex of the left ventricle and the animal was perfused with $10 \mathrm{ml}$ of PBS to eliminate the blood. Perfusion was continued with $5 \mathrm{ml}$ of PBS containing either LIBSMPIO or control-MPIO $\left(1.5 \times 10^{8} \mathrm{MPIO} / \mathrm{ml}\right.$ for each, corresponding to approximately $6.5 \mathrm{mg}$ iron $/ \mathrm{kg}$ body weight): 7 mice were perfused with LIBS-MPIO and 6 with control MPIO via the left ventricle. After $30 \mathrm{~min}$, mice were again perfused under physiological pressure with $10 \mathrm{ml}$ of PBS followed by $5 \mathrm{ml}$ of $4 \%$ paraformaldehyde containing $2 \mathrm{mM}$ gadoteridol (Prohance, Bracco, UK).

After perfusion, the skin was removed, the leg with the area of injury cut, kept in $4 \%$ paraformaldehyde/ $2 \mathrm{mM}$ gadoteridol for $24 \mathrm{~h}$ and then embedded in a glass MR tube containing $2 \%$ high-grade, low-melting-point agarose (Cambrex, Rockland, Me., USA).

\section{Ex vivo MRI}

Ex vivo MRI was performed at $11.7 \mathrm{~T}$ (Bruker BioSpin 500 $\mathrm{MHz}$, Bruker, Coventry, UK) using a $13-\mathrm{mm}^{1} \mathrm{H}$ birdcage radiofrequency coil (RAPID Biomedical, Würzburg, Germany). A 3D gradient echo sequence $(\mathrm{TE}=4 \mathrm{~ms} / \mathrm{TR}=90 \mathrm{~ms}$, field of view $13 \times 13 \times 19.5 \mathrm{~mm}$, matrix size $256 \times 256 \times 384$, two averages, imaging time $\sim 7 \mathrm{~h}$ /sequence) was used in an unattended overnight run. The MRI sequence employed provided $\mathrm{T}_{2}$-weighted images that are typically used for the identification of iron-based contrast agents [19]. Data reconstruction was performed off-line with a final isotropic resolution of $25 \mu \mathrm{m}$.

\section{Histology and Quantification of MPIO Binding in the Injured Femoral Artery}

After MRI, specimens were decalcified in $10 \%$ formic acid overnight, dehydrated through graded ethanol solutions and Neo-clear (VMR, UK), paraffin embedded and serially sectioned (8 $\mu \mathrm{m}$ thick). Specimens were stained for iron (Accustain, Sigma) referring to the manufacturer's protocol. The number of conjugated MPIO bound to the injured luminal vessel wall was quantified and averaged in 20-25 sections from the injured vessel per animal site using light microscopy.

For platelet visualization with immunohistochemistry, deparaffinized and rehydrated sections were saturated in $1 \% \mathrm{H}_{2} \mathrm{O}_{2}$ for $20 \mathrm{~min}$, added to simmering citrate buffer and boiled for $4 \mathrm{~min}$ in a pressure cooker for antigen retrieval. Specimens were washed in PBS/Tween-20, incubated with protein block solution (DakoCytomation, Hamburg, Germany) for $4 \mathrm{~h}$ and incubated overnight at $4^{\circ} \mathrm{C}$ with rat anti-mouse CD61 antibody (1:8,000, InterCell Technologies, Jupiter, Fla., USA). After washing with PBS, a biotinylated goat anti-rat IgG (1:200, Vector, Burlingame, Calif., USA) secondary antibody was used. Slides were washed with PBS, and a peroxidase reaction was performed using Vectastain RTU Elite ABC reagent and Vector NovaRed (both Vector). Finally, sections were dehydrated and mounted with Permount (Biomeda, Foster City, Calif., USA), and MPIO binding to platelets was evaluated on a light microscope.

MPIO Binding in the Femoral Artery by ex vivo MRI

Quantification of MPIO binding was performed blind to the origin of the image. Antibody-conjugated MPIO binding was defined as an intense circular signal void (appearing as black circular areas, marked with arrows in fig. $2 \mathrm{a}$ ) on the luminal surface of the femoral artery in $\geq 2$ consecutive slices. MPIO appearing in multiple sections were counted only once. The region of interest in each animal started directly proximal to the perforating branch of the femoral artery, and MPIO-induced signal void was quantified in 150 consecutive slices for each artery. MPIO binding is presented as low-signal areas per artery. Segmented images were reconstructed in three dimensions using the 3D Constructor plug-in for ImagePro Plus to visualize the distribution of MPIO binding through- 
out the femoral artery, with the low-signal area assigned to the red channel and the anatomical image to the green channel.

\section{Biodistribution of MPIO in vivo}

Live mice ( $n=3$ per group) were injected with LIBS-MPIO via the tail vein in a dose of $4 \times 10^{8} \mathrm{MPIO}$ in total $(4.5 \mathrm{mg}$ iron $/ \mathrm{kg}$ body weight), since we successfully applied this dose of MPIO in previous experiments of in vivo VCAM imaging [20, 21]. One group of animals was sacrificed after $30 \mathrm{~min}$, and the other group after $24 \mathrm{~h}$. Frozen sections of lungs, liver, kidneys and spleen were obtained (slice thickness $10 \mu \mathrm{m}$ ) using a standard protocol. MPIO distribution was quantified on a microscope in five high-power $(100 \times)$ fields of view per organ to obtain a mean for each animal. No staining of the sections was necessary, since MPIO could be easily identified in tissues due to their typical appearance.

\section{Statistical Methods}

Data are expressed as means \pm SEM. Parametric data were compared using t tests. Statistical significance was assigned to $\mathrm{p}<0.05$

\section{Results}

\section{LIBS-MPIO Detect Activated GPIIb/IIIa Receptors on} Platelets

In figure 1a, layers of human platelet aggregates labelled with anti-P-selectin antibody show bright green fluorescence. Superimposed on these platelet aggregates are red particles, corresponding to fluorescent LIBSMPIO (online version). In figure $1 \mathrm{~b}$, these MPIO are confined to the platelet aggregates without non-specific background retention. By contrast, there is absence of binding of control MPIO conjugated to an irrelevant single-chain antibody. In figure 1c, the $\mathrm{z}$-stack reconstruction in confocal microscopy shows LIBS-MPIO binding (red, online version) to P-selectin-stained platelets (green, online version), emphasizing their relative size and spatial relations and the presence of multiple LIBS-MPIO on platelet clusters.

\section{LIBS-MPIO Bound to Wall-Adherent Platelets}

Detected by ex vivo MRI

Unilateral femoral artery wire injury was performed in mice without complication. One control animal was excluded from the quantification analysis, due to marked variation in the MRI quantification of MPIO between two observers.

Ex vivo $\mathrm{T}_{2}$-weighted MRI of injured arterial segment typically demonstrated intrinsic multiple low-signal areas within the arterial wall (fig. 2b). In contrast, circular intense signal voids emerged within the vessel lumen, adjacent to the vessel wall. In some cases, the 'bloom' effect
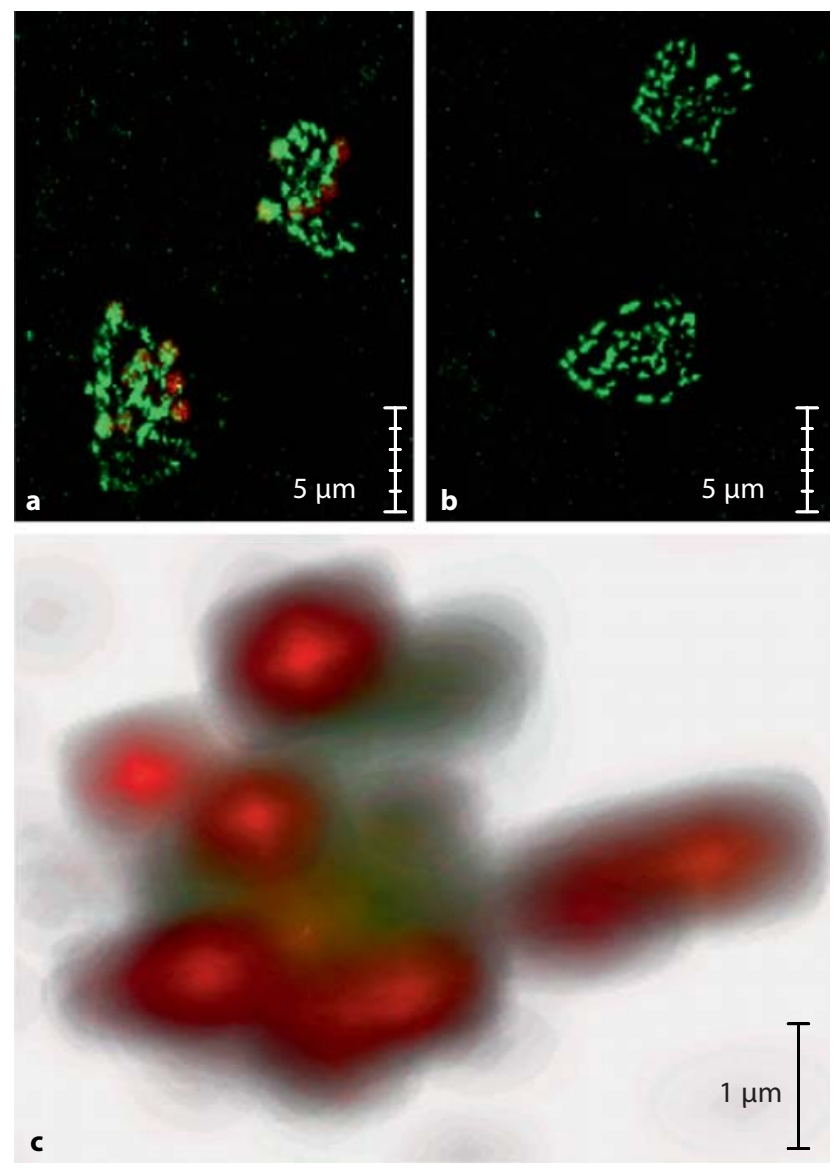

Fig. 1. Confocal microscopy of human platelets immobilized on fibrinogen and detected by anti-CD62 antibody, shown with avidin-fluorescein immunofluorescence (a-c: green in the online version). Platelets incubated with the autofluorescent LIBS-MPIO contrast agent (a, c: red in the online version) show specific binding to platelets (a). Incubation of platelets with control-MPIO contrast agent shows no MPIO binding (b). 3D rendering of stained platelets incubated with LIBS-MPIO: multiple LIBSMPIO binding to clusters of activated platelets via GPIIb/IIIa (c).

due to distortion of the local magnetic field extended far beyond the physical space occupied by MPIO, and caused encroachment of the contrast effect into the vessel wall. These features were observed in the wire-injured arteries of all mice injected with LIBS-MPIO (fig. 2a). In quantitative analysis, luminal areas of low signal, suggesting MPIO accumulation, were $\sim 4$-fold higher in LIBSMPIO-injected animals than in control-MPIO-perfused animals (values given as total number of round circular signal void per animal; 23.72 vs. 6.2 , p $<0.01$; fig. $2 \mathrm{~d}$ ).

MPIO binding was confirmed in histology. MPIO have a typical appearance under the microscope as artificially round structures. Depending upon the focus used 

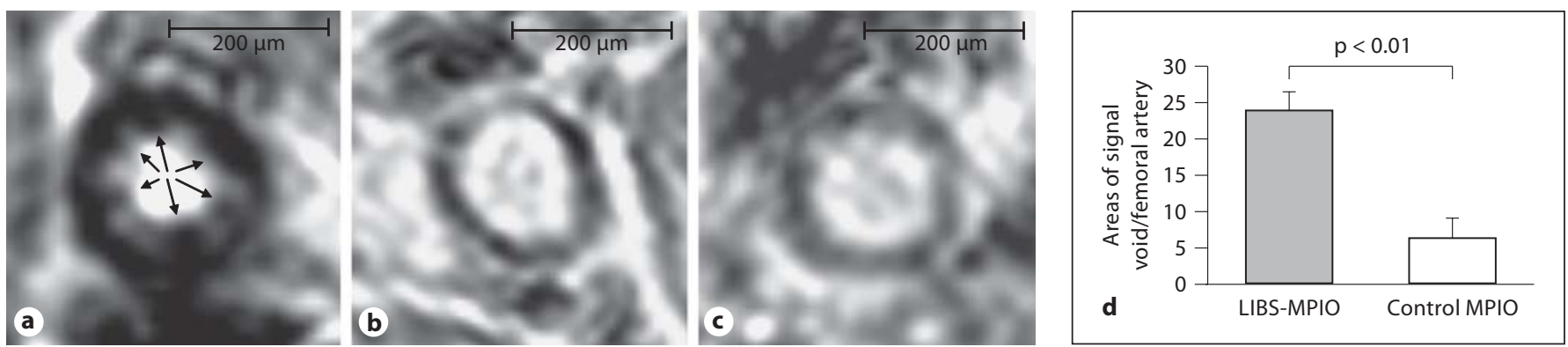

Fig. 2. Ex vivo MRI at 11.7 T. 3D gradient echo sequence $(\mathrm{TR}=90$ $\mathrm{ms}, \mathrm{TE}=4 \mathrm{~ms}$, imaging time $7 \mathrm{~h}$ and resolution $25 \mu \mathrm{m}$ isotropic). a The injured femoral artery exposed to LIBS-MPIO shows multiple, intensely-low-signal, lobulated areas at the interface between vessel wall and lumen (arrows). In the injured artery perfused with control-MPIO (b), there is a small amount of inherent signal heterogeneity, but this is clearly distinguished from the ap-

pearance of bound contrast (a). The same is true for the example of an uninjured artery without MPIO perfusion, which yields signal effects comparable to the injured vessel in $\mathbf{b}$. Areas of signal void were quantified in total for every artery (d). Quantification of MPIO-induced MRI signal voids revealed a significant difference between LIBS-MPIO- and control-MPIO-perfused mice $(\mathrm{p}<0.05)$.

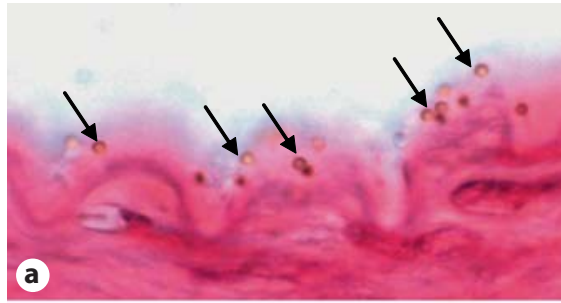

on the microscope, they can appear as round structures with a green, blue or red center and a halo of brown or black color (fig. $3 \mathrm{a}-\mathrm{c}$, online version). The MPIO were clearly distinguishable from natural tissue structures or wall-adherent cells (fig. 3a, c), and staining for iron was negative. The absence of specific iron staining can be attributed to the impervious polystyrene shell that effectively insulated the iron oxide core. This effect of negative iron stain of MPIO was unexpected, however, we decided to continuously apply iron stain to our sections as tissue structures were easily detectable, and as we were simultaneously able to exclude other iron accumulations than MPIO responsible for our signal effect (clots of red blood cells).

MPIO quantification on the vessel surface of LIBSMPIO- and control-MPIO-perfused animals showed a significantly higher binding for LIBS-MPIO compared to control MPIO (9.98 vs. $0.5 \mathrm{MPIO} /$ section, p<0.01; fig. 3a,

Fig. 3. Histology of representative injured femoral artery segments. a Multiple MPIO binding to the injured wall after perfusion with LIBS-MPIO (arrows). b In contrast, no binding can be observed after control-MPIO perfusion. c The arterial wall and surrounding tissue appears red (online version) due to the pararosaniline stain contained in the standard protocol of iron staining. Co-localization of LIBS-MPIO contrast agent and platelets was confirmed using immunohistochemistry for CD61; single platelets can be seen as rust-colored (online version) structures with NovaRed (marked with arrows). d Quantification of MPIO bound per section in LIBS-MPIO- and control-MPIO-perfused mice, showing highly specific binding of the LIBS-targeted contrast agent $(\mathrm{p}<0.01)$. 


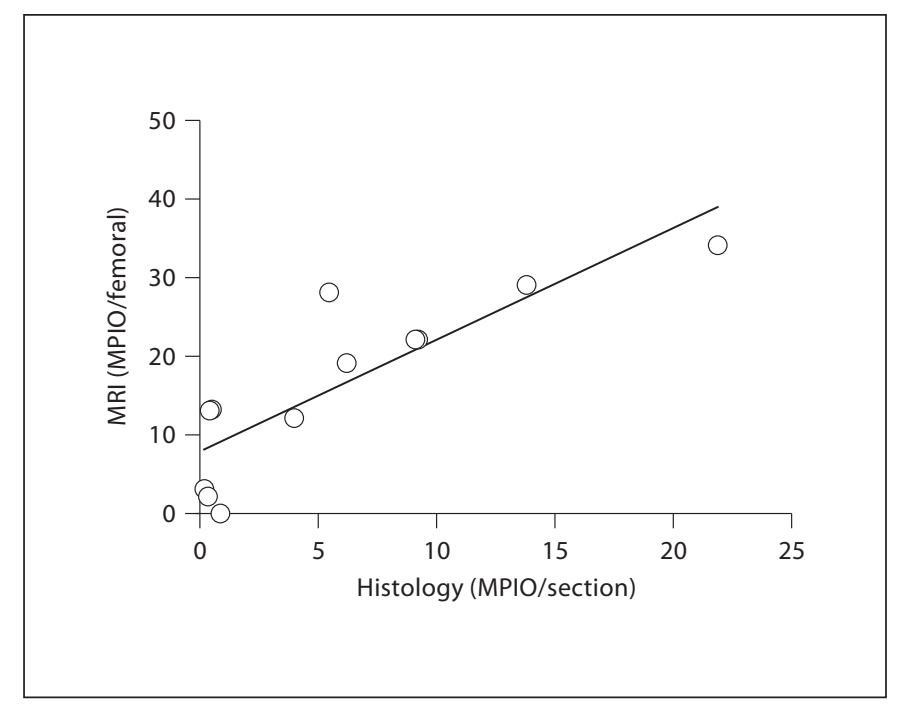

Fig. 4. Quantification of MPIO binding. Quantification of MPIO signal per injured leg by MRI and histological analysis were strongly correlated $\left(\mathrm{R}^{2}=0.72 ; \mathrm{p}<0.001\right)$.

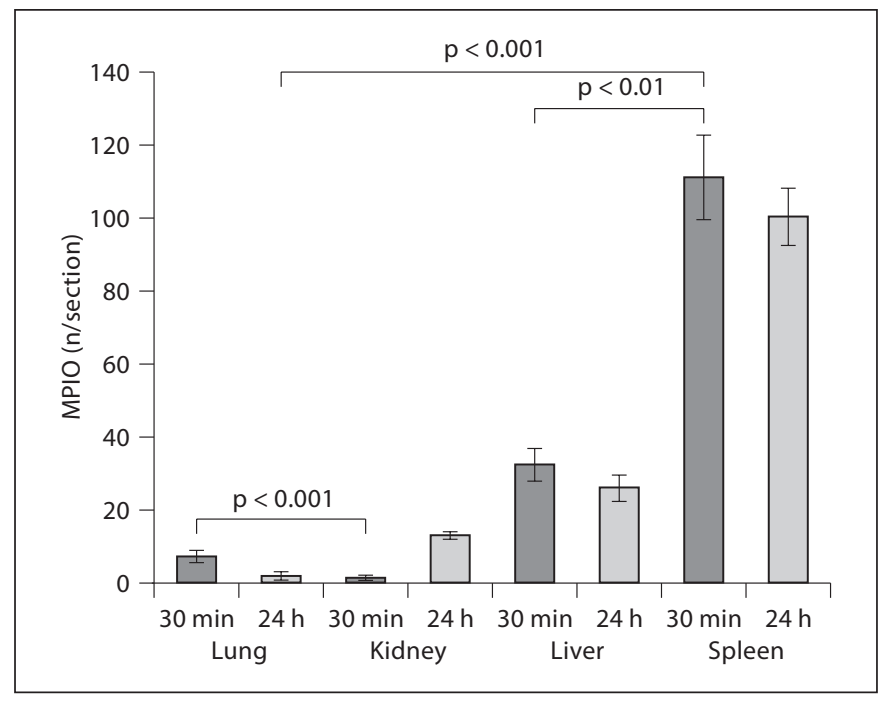

Fig. 6. In vivo biodistribution. MPIO were quantified in the lung, kidney, liver and spleen, $30 \mathrm{~min}$ and $24 \mathrm{~h}$ after intravenous administration. Retention by the lungs is minimal at either time point. By contrast, there was rapid uptake by the spleen and liver. Efficient removal of contrast agent from the circulation is advantageous for in vivo imaging, where background blood MPIO may otherwise obscure specifically bound contrast.

Fig. 5. Three-dimensional reconstruction of the femoral artery of an anti-LIBSMPIO-injected mouse. (Online version only: a In this reconstruction, the anatomical image has been allocated to the green channel. Therefore, the femoral artery lumen appears as a green tube. b Segmented regions of contrast were allocated to the red channel). c The merged image shows diffuse and relatively even MPIO binding along the luminal surface of the injured vessel segment. The magnetic field distortion of MPIO reaches beyond their physical diameter, therefore MPIO contrast effects extend into the vessel wall.
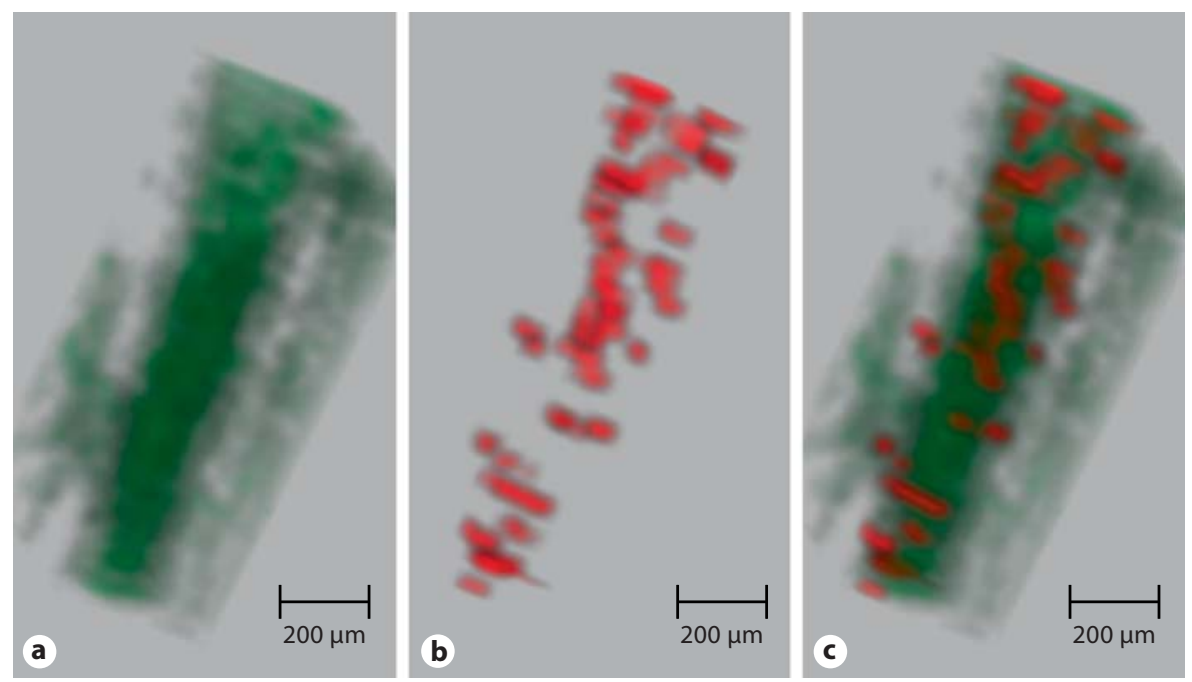

b, d). Co-localization of LIBS-MPIO and platelets adherent to the arterial wall was confirmed by immunostaining for the platelet marker CD61 (fig. 3c).

Analysis of MPIO quantification in histology compared to quantification by ex vivo MRI revealed a strong correlation $\left(\mathrm{R}^{2}=0.7219, \mathrm{p}<0.001\right.$; fig. 4). Therefore, MPIO signal quantity, determined by MRI, directly re- flected the quantity of MPIO bound to the injured vessel wall. To appreciate the binding patterns of MPIO within an injured segment, 3D reconstruction of segmented images was undertaken. In figure 5, structures appearing as MPIO-induced signal void were encoded by a red color, and for the vessel lumen a green color was chosen (online version). Bright red spots correspond to areas of intense 
low signal and are seen scattered throughout this injured arterial segment. As the MPIO contrast effect extends beyond the physical limit of the particle $(\sim 50$ times the physical diameter), it appears that some red spots are partially located within, even beyond, the vessel wall (online version). Furthermore, it is possible that not every spot represents a single MPIO, but can also be caused by colocalized MPIO on a platelet surface.

\section{Biodistribution of MPIO in vivo}

All mice tolerated MPIO injection and showed no signs of ill health up to $24 \mathrm{~h}$ after injection. MPIO were identified in the lung, kidney, liver and spleen after 30 min and $24 \mathrm{~h}$ (fig. 6). Even at the early time points, there was minimal retention by the lungs (at $30 \mathrm{~min}$, lung: 6.9 MPIO/section vs. spleen: $110 \mathrm{MPIO} /$ section; $\mathrm{p}<0.001$ ). As expected, the bulk of the MPIO load was rapidly sequestered by the liver and the spleen (liver: $31.8 \mathrm{MPIO} /$ section; spleen: $110 \mathrm{MPIO} /$ section; $\mathrm{p}<0.001$ vs. MPIO/ section in the lung and kidneys at $30 \mathrm{~min}$ ).

\section{Discussion}

Imaging platelet thrombi is of considerable interest to aid in diagnosis and guide therapy in acute vascular syndromes. We report the development and application of a molecular contrast agent that targets activated platelets and enables quantification of contrast binding using MRI. We have used a single-chain antibody that recognizes only the active conformation of GPIIb/IIIa, and therefore confers both molecular and functional specificity. To the best of our knowledge, this is the first report on functional MRI of a cellular surface molecule according to its conformational state. Secondly, we have exploited the fact that intravascular structures are accessible to $1-\mu \mathrm{m}$-sized particles that are orders of magnitude larger than the iron oxide nanoparticles used previously for similar applications. This approach has provided exceptionally conspicuous contrast on MRI.

As no reliable animal model of plaque rupture exists, we used mouse femoral wire injury to induce platelet thrombi. The time course of cellular events following femoral wire injury in this model is well described and consistently shows confluent platelet deposition on the denuded endothelium after $24 \mathrm{~h}$ (fig. 3c) [18]. We used this as the basis for targeting activated platelets with single-chain antibodies against LIBS on the activated GPIIb/IIIa receptor. This antibody confers functional specificity since its binding is dependent upon the pres- ence of the activated form of GPIIb/IIIa [17]. These properties make LIBS a unique target to mediate MPIO attachment to platelet thrombi. The targeting potency of the anti-LIBS single-chain antibody has been recently demonstrated by the strong enrichment of an anticoagulant effector molecule at clots [16]. Following left-ventricular injection, LIBS-MPIO bound specifically to platelet thrombi in the femoral artery. Using a $\mathrm{T}_{2}$-weighted MRI sequence, signal voids corresponding to MPIO binding were quantified. The extent of signal extinction caused by the MPIO in $\mathrm{T}_{2}$-weighted MRI corresponded to the MPIO number on histology.

A limitation of the current study is the absence of in vivo imaging. Following intravenous injection, we have not been able to demonstrate accumulation of LIBSMPIO on the walls of the injured femoral artery (data not shown). This may be explained by the type of injury model used in our study. The model of femoral artery wire injury is known to result in a thin monolayer of platelets on the injured arterial wall $[18,22]$. Thus, only a few targeting epitopes are exposed. Furthermore, it is known that arterial shear stress in the femoral artery is high and thus the limited number of epitopes may not be sufficient to enable adhesion of the $1-\mu \mathrm{m}$ MPIO. It is also known from leukocyte biology that to effect binding to the endothelium under flow conditions is a complex biological task requiring the interaction of multiple receptors [2325]. Indeed, single ligand binding to either VCAM-1 or to P-selectin resulted in modest retention of MPIO, which was markedly increased by the combination of ligands against both VCAM-1 and P-selectin [20]. However, the application of MPIO-based contrast agents is a promising strategy for in vivo imaging, as we recently showed by targeting VCAM in acute brain inflammation with a VCAM-targeted MPIO [21]. Therefore, the size of the MPIO does not seem to be a limitation for the successful binding of the contrast agent to the epitope, but the model of a platelet monolayer applied in this study is suboptimal. Thus, while the current experiments show proof of principle, limitations of the specific model mean that in vivo imaging remains to be demonstrated, potentially in a different vessel with larger thrombi.

Previous targeted contrast agent approaches have included integrin-targeted gadolinium-rich perfluorocarbon nanoparticles [26], peptide-conjugated nanoparticles of iron oxide [27] and fibrin-specific cyclic peptides labelled with gadolinium [13]. However, the quantity of contrast agent that can be delivered, and therefore the intensity of contrast effect achieved, is relatively limited, particularly for low-abundance targets. Iron micropar- 
ticles have been used for cellular imaging applications in transplant rejection [28] and for tracking during embryogenesis [15]. We have recently demonstrated the use of MPIO for molecular imaging in mice in vivo. MPIO directed against VCAM-1 identified acute brain inflammation with high specificity and conspicuity [21]. Building on the success of this approach, we have extended the use of MPIO to image platelet thrombi. MPIO carry a high payload of contrast that is not readily dispersed. Unlike ultrasmall particles of iron oxide, MPIO binding is readily distinguished from inherent signal heterogeneity in the vessel wall providing excellent visualization by MRI.

A strength of MPIO use for in vivo imaging is their rapid clearance from the blood phase. Our data show that binding to the target is rapid. In order to achieve good contrast from background, it is essential that MPIO are rapidly cleared from the circulation. In vivo biodistribution experiments reported here show rapid sequestration in the spleen. Importantly for intravenously administered particles, we see very little retention in the lungs and no sign of microscopically visible tissue injury, e.g. organ infarcts or clotting, inside the vessels.

As demonstrated here, combining imaging and single-chain antibody technologies can provide a powerful approach to generate a functionally specific molecular imaging probe. An important consideration is potential immunogenicity of the contrast agent. Use of singlechain antibodies, consisting only of the variable regions, minimizes this effect [29]. Single-chain antibodies, in general, are a good basis for the production of contrast agents. These recombinant antibody fragments can be produced in bacteria at low cost and are easily amenable to commercial scale-up. Furthermore, the possibility to add functional groups allows for high-purity production using affinity columns [30].

In this study, we used commercially available MPIO for targeting histidine-tagged single-chain antibody. The polystyrene coating of these specific particles is not suitable for use in humans due to their potential toxicity. However, work is ongoing on the development of micronsized biodegradable contrast particles for clinical use [3133].

\section{Conclusion}

We have shown that anti-LIBS-MPIO complexes can be used to target activated platelets deposited on injured vessel walls in mice. We have exploited endovascular accessibility to use micron-sized contrast agents that convey a substantial contrast payload. We have used a newly developed single-chain antibody with specificity for the ligand-bound GPIIb/IIIa receptor. This confers functional specificity to image activated platelets adhering to the vessel wall and thus may provide a valuable opportunity to identify unstable atherosclerotic plaques with MRI.

\section{Acknowledgments}

The authors gratefully acknowledge the contribution of Mr. Phil Townsend, Ms. Julia Moeller and Ms. Irene Neudorfer. We thank Dr. Susan Smyth (Chapel Hill, N.C., USA) for donating the anti-P-selectin antibody. Prof. Kieran Clarke is thanked for enabling the MRI studies.

The study was supported by the Wellcome Trust (London, UK) and the National Heart Foundation (Australia).

\section{References}

1 Falati S, Gross P, Merrill-Skoloff G, Furie BC, Furie B: Real-time in vivo imaging of platelets, tissue factor and fibrin during arterial thrombus formation in the mouse. Nat Med 2002;8:1175-1181

2 Burke AP, Farb A, Malcom GT, Liang YH, Smialek J, Virmani R: Coronary risk factors and plaque morphology in men with coronary disease who died suddenly. N Engl J Med 1997;336:1276-1282.

$>$ Sabatine MS, Cannon CP, Gibson CM, Lopez-Sendon JL, Montalescot G, Theroux P, et al: Addition of clopidogrel to aspirin and fibrinolytic therapy for myocardial infarction with ST-segment elevation. N Engl J Med 2005;352:1179-1189.

\footnotetext{
4 Randomised trial of intravenous streptokinase, oral aspirin, both, or neither among 17,187 cases of suspected acute myocardial infarction: ISIS-2. ISIS-2 (Second International Study of Infarct Survival) Collaborative Group. Lancet 1988;2:349-360.

5 Yusuf S, Zhao F, Mehta SR, Chrolavicius S, Tognoni G, Fox KK: Effects of clopidogrel in addition to aspirin in patients with acute coronary syndromes without ST-segment elevation. N Engl J Med 2001;345:494-502.

-6 Montalescot G, Barragan P, Wittenberg O, Ecollan P, Elhadad S, Villain P, et al: Platelet glycoprotein IIb/IIIa inhibition with coronary stenting for acute myocardial infarction. N Engl J Med 2001;344:1895-1903.
}

7 Use of a monoclonal antibody directed against the platelet glycoprotein IIb/IIIa receptor in high-risk coronary angioplasty. The EPIC Investigation. N Engl J Med 1994; 330:956-961.

8 Mehta SR, Yusuf S, Peters RJ, Bertrand ME, Lewis BS, Natarajan MK, et al: Effects of pretreatment with clopidogrel and aspirin followed by long-term therapy in patients undergoing percutaneous coronary intervention: the PCI-CURE study. Lancet 2001; 358:527-533

$\checkmark 9$ Mizuno K, Satomura K, Miyamoto A, Arakawa K, Shibuya T, Arai T, et al: Angioscopic evaluation of coronary-artery thrombi in acute coronary syndromes. N Engl J Med 1992;326:287-291. 
10 Choudhury RP, Fuster V, Fayad ZA: Molecular, cellular and functional imaging of atherothrombosis. Nat Rev Drug Discov 2004;3: 913-925.

-11 Jaffer FA, Libby P, Weissleder R: Molecular and cellular imaging of atherosclerosis: emerging applications. J Am Coll Cardiol 2006;47:1328-1338.

-12 Flacke S, Fischer S, Scott MJ, Fuhrhop RJ, Allen JS, McLean M, et al: Novel MRI contrast agent for molecular imaging of fibrin: implications for detecting vulnerable plaques. Circulation 2001;104:1280-1285.

- 13 Botnar RM, Perez AS, Witte S, Wiethoff AJ, Laredo J, Hamilton J, et al: In vivo molecular imaging of acute and subacute thrombosis using a fibrin-binding magnetic resonance imaging contrast agent. Circulation 2004; 109:2023-2029.

-14 Flaumenhaft R, Tanaka E, Graham GJ, De Grand AM, Laurence RG, Hoshino K, et al: Localization and quantification of plateletrich thrombi in large blood vessels with near-infrared fluorescence imaging. Circulation 2007; 115:84-93.

-15 Shapiro EM, Skrtic S, Sharer K, Hill JM, Dunbar CE, Koretsky AP: MRI detection of single particles for cellular imaging. Proc Natl Acad Sci USA 2004;101:10901-10906.

- 16 Stoll P, Bassler N, Hagemeyer CE, Eisenhardt SU, Chen YC, Schmidt R, et al: Targeting ligand-induced binding sites on GPIIb/IIIa via single-chain antibody allows effective anticoagulation without bleeding time prolongation. Arterioscler Thromb Vasc Biol 2007;27:1206-1212.

-17 Schwarz M, Katagiri Y, Kotani M, Bassler N, Loeffler C, Bode C, et al: Reversibility versus persistence of GPIIb/IIIa blocker-induced conformational change of GPIIb/IIIa $\left(\alpha_{\text {IIB }} \beta_{3}\right.$, CD41/CD61). J Pharmacol Exp Ther 2004; 308:1002-1011.
18 Roque M, Fallon JT, Badimon JJ, Zhang WX, Taubman MB, Reis ED: Mouse model of femoral artery denudation injury associated with the rapid accumulation of adhesion molecules on the luminal surface and recruitment of neutrophils. Arterioscler Thromb Vasc Biol 2000;20:335-342.

19 Weisskoff RM, Caravan P: MR contrast agent basics; in Lardo AC, Fayad ZA, Fuster V, Chronos N (eds): Cardiovascular Magnetic Resonance. Established and Emerging Applications. London, Dunitz, 2003.

20 McAteer MA, Schneider JE, Ali ZA, Warrick N, Bursill CA, von zur Muhlen C, et al: Magnetic resonance imaging of endothelial adhesion molecules in mouse atherosclerosis using dual-targeted microparticles of iron oxide. Arterioscler Thromb Vasc Biol 2007; 28:77-83.

21 McAteer MA, Sibson NR, von zur Muhlen C, Schneider JE, Lowe AS, Warrick N, et al: In vivo magnetic resonance imaging of acute brain inflammation using microparticles of iron oxide. Nat Med 2007;13:1253-1258.

$>22$ Lindner V, Fingerle J, Reidy MA: Mouse model of arterial injury. Circ Res 1993;73: 792-796.

23 Ramos CL, Huo Y, Jung U, Ghosh S, Manka DR, Sarembock IJ, et al: Direct demonstration of P-selectin- and VCAM-1-dependent mononuclear cell rolling in early atherosclerotic lesions of apolipoprotein E-deficient mice. Circ Res 1999;8:1237-1244.

24 Bhatia V, Bhatia R, Dhindsa S, Dhindsa M: Imaging of the vulnerable plaque: new modalities. South Med J 2003;96:1142-1147.

25 Eniola AO, Willcox PJ, Hammer DA: Interplay between rolling and firm adhesion elucidated with a cell-free system engineered with two distinct receptor-ligand pairs. Biophys J 2003;85:2720-2731.
26 Winter PM, Morawski AM, Caruthers SD, Fuhrhop RW, Zhang H, Williams TA, et al: Molecular imaging of angiogenesis in earlystage atherosclerosis with $\alpha_{\mathrm{V}} \beta_{3}$-integrintargeted nanoparticles. Circulation 2003; 108:2270-2274.

-27 Kelly KA, Allport JR, Tsourkas A, ShindePatil VR, Josephson L, Weissleder R: Detection of vascular adhesion molecule-1 expression using a novel multimodal nanoparticle. Circ Res 2005;96:327-336.

28 Wu YL, Ye Q, Foley LM, Hitchens TK, Sato $\mathrm{K}$, Williams JB, et al: In situ labeling of immune cells with iron oxide particles: an approach to detect organ rejection by cellular MRI. Proc Natl Acad Sci USA 2006;103: 1852-1857.

29 Das D, Kriangkum J, Nagata LP, Fulton RE, Suresh MR: Development of a biotin mimic tagged $\mathrm{ScFv}$ antibody against western equine encephalitis virus: bacterial expression and refolding. J Virol Methods 2004;117:169177.

30 Schwarz M, Meade G, Stoll P, Ylanne J, Bassler N, Chen YC, et al: Conformationspecific blockade of the integrin GPIIb/IIIa: a novel antiplatelet strategy that selectively targets activated platelets. Circ Res 2006;99: 25-33.

31 Zhu D, White RD, Hardy PA, Weerapreeyakul N, Sutthanut K, Jay M: Biocompatible nanotemplate-engineered nanoparticles containing gadolinium: stability and relaxivity of a potential MRI contrast agent. J Nanosci Nanotechnol 2006;6:996-1003.

32 Hamoudeh M, Fessi H: Preparation, characterization and surface study of poly-epsilon caprolactone magnetic microparticles. J Colloid Interface Sci 2006;300:584-590.

33 Chen HH, Le Visage C, Qiu B, Du X, Ouwerkerk R, Leong KW, et al: MR imaging of biodegradable polymeric microparticles: a potential method of monitoring local drug delivery. Magn Reson Med 2005;53:614620 . 